\title{
Low Light Energy Autonomous LoRaWAN Node
}

\author{
Marcel Meli ${ }^{1}$, Manuel Brütsch ${ }^{1}$, Stefan Stajic ${ }^{1}$, Manuel Böbel ${ }^{1}$, David Lorenz ${ }^{1}$ \\ Lukas Hegetschweiler ${ }^{2}$ \\ Dimitris Karanassos ${ }^{3}$, Charalampos Kouzinopoulos ${ }^{3}$ \\ ${ }^{1}$ ZHAW, Technikumstrasse 9, 8401 Winterthur, Switzerland, \\ \{marcel.meli, manuel.bruesch, stefan.stajic, manuel.boebel, david.lorenz\}@zhaw.ch, \\ https://www.zhaw.ch \\ ${ }^{2}$ Breitensteinstrasse 90, 8037 Zürich, Switzerland, hegetschweiler.lukas@gmail.com \\ ${ }^{3}$ CERTH, 6th Klm. Charilaou - Thermi Road, 57001 Thermi, Thessaloniki, Greece, \\ \{dkaranassos, kouzinopoulos\}@iti.gr, https://www.iti.gr/iti/index.html
}

\begin{abstract}
A LoRaWAN node powered using an $8 \mathrm{~cm}^{2}$ solar cell was designed and its low light harvesting performance evaluated. The embedded system is used to sense some parameters and transmit the results every 10 minutes, using the spreading factor SF7BW125 and transmitting with $+8 \mathrm{dBm}$, which allows the coverage of a small building. The node can cold start with less than 30 lux. Once started, its operations can be sustained down to 20 lux. Operation at higher spreading factors or higher RF output power is also possible if the transmission interval is increased. Such a performance enables the use of energy autonomous LPWAN nodes in poorly lit environments. The small size of the solar cell makes it possible to build small nodes.
\end{abstract}

Keywords-energy harvesting; solar cells; LPWAN; LoRaWAN; power management

\section{INTRODUCTION AND MOTIVATION}

In the last decade, Low Power Wide Area Networks (LPWAN) have become a popular research topic and new application fields have been introduced such as multinode, large-scale and smart agriculture implementations [1]-[5]. Most of the research defines basic parameters and requirements on LPWAN such as ease of installation, low price, minimum maintenance, small surface of nodes in the network and low energy consumption [6]-[12]. The aforementioned systems take advantage of sensor nodes that can transmit data over long distances, with a range of up to 30 kilometers and with a low power consumption [6]. Most systems are battery powered. There are several issues that might arise from such implementations, such as battery failures due to environmental changes or due to the aging effect of the electrochemical structure of the battery. To overcome these issues, the scientific community has been looking into solutions that are based on renewable energy.

Solar cells are practical and probably one of the most used harvesters. Solar calculators and even watches are now among the products where such cells are used to improve energy autonomy. They are also popular when considering the energy autonomy of IoT nodes [13]-[20]. They can be used alone or combined with storage elements. Solar cells have also been explored for products where short-range communication is needed. In several cases, they are employed outdoor, where illuminance levels are high. Using solar energy in LPWAN nodes introduces additional challenges, especially in environments where light intensity is low. This has to do with the amount of energy required by such nodes and with the performance of the power management. Whereas WPAN systems such as those based on Bluetooth Smart can advertise with tens of microjoules, tens or even hundreds of millijoules are required for LoRa or Sigfox LPWAN nodes transmitting with maximum RF power [11], [19]. That means harvesting longer or using larger solar cells, which is not very practical indoors. Moreover, if the office is not lit, there is no energy to be harvested, meaning that the nodes need to store enough energy for the "bad times". In cases where there is not enough light for several days, the storage will be depleted, unless elements with larger capacities are used. The time required to recharge the storage to the point that the node can be used is an important criterion. A system that can run in low light conditions has several advantages:

- It can be used in environments that are poorly lit.

- It can help reduce the constraints on other parts of the architecture (e.g. storage elements).

In order to meet some of the challenges mentioned above, an existing low-power LoRaWAN of the ZHAWInES was modified in this work ${ }^{1}$ to allow it to run on solar energy. That serves as an early verification test in the AMANDA project. The performance of the node in a low light environment was then evaluated in an office, with

${ }^{1}$ This work is the result of an internal project of the ZHAW Institute of Embedded System with some aspects related to the AMANDA Project sponsored by the Horizon2020 program under the grant agreement ID: 825464. ZHAW and CERTH are part of the AMANDA Consortium. The solar cell used is from Lightricity, a firm that is also part of the AMANDA consortium. 
the emphasis on low power consumption and the use of a high efficiency solar cell now available on the market.

\section{StATE OF THE ART AND Key ACHIEVEMENTS}

In order to safeguard the system's performance and increase the lifetime of energy autonomous systems, the power source has been combined with various storage elements. These can be supercapacitors [8] or small backup batteries [9],[10]. This has encouraged a tendency for alternative power sources that use renewable energy to enable LPWAN systems to expand their life cycle and ensure their power autonomy. There has also been a focus on combining solar harvesting with other techniques like wireless charging [7], incorporating batteries or supercapacitors [9] or differentiating the platform's architectural structure [12] to ensure a proper working environment for such devices. To that end, more and more studies try to integrate harvesters that achieve lower illuminance thresholds for their system to operate as intended [8], [10], [12], [16]-[20]. In most cases, the node is used outdoor, where there is often enough light. Systems that work indoor tend to concentrate on light conditions in a lit office (above 200 lux) as the lower limit. To the best of the authors' knowledge at the time of this paper's submission, there has been no reported study for LPWAN nodes with a minimum illuminance below 100 lux. The key achievements of this work are:

- A Low-power LoRaWAN node that can work below 25 lux and transmit frames using LoRaWAN with SF7BW125 every 10 mins.

- A node that can cold start with an illuminance of 30 lux.

- A node with a powerful Cortex-M4 compatible processor working under low light conditions.

- Additionally, the solution uses an external FRAM and optimises the energy harvesting process by switching off the embedded system between measurements. This is done in order to limit the load on the storage elements while harvesting. A similar strategy was described in [19].

- The node can easily be modified to increase the communication range, by changing the storage element and programming the radio to work with a higher spreading factor.

\section{DESCRIPTION OF THE NODE}

Fig. 1 shows the block diagram of the node. Energy efficiency is achieved by using fitting components and an appropriate strategy.

\section{A. Components}

The main elements of the node are succinctly described below.

- The solar cell is the ExCellLight EXL10-4V170 module from Lightricity [21]. Its power density for the active area is $21.8 \mu \mathrm{W} / \mathrm{cm} 2$ at 200 lux under CCFL spectrum $(18.2 \mu \mathrm{W} / \mathrm{cm} 2$ at 200 lux under LED spectrum). The basic cells are connected such as to deliver a voltage that is already sufficient for the electronics (4 volts), obsoleting the need of a booster.

- The power management (PM or PMIC) is the EM8502 from EM Microelectronic [22]. This is a complex and versatile device that can be configured via on-chip EEPROM or RAM in order to optimise the power management functions. The device supports up to 2 storage elements called Long Term Storage (LTS) and Short Term Storage (STS). They can be used to allow both fast start-up of the load (STS) and long operation (LTS). Several comparators with programmable levels help control the use of STS and LTS (charge, discharge, protection limits). The available energy is released at precise voltages, depending on the comparator settings. Some of the parameters of the PM can be controlled on-the-fly by a microcontroller via the serial interface. The device also includes a low energy timer to schedule the release of the energy.

- The storage. In this work, a $10 \mathrm{mF}$ supercapacitor is used as storage element. It accumulates energy under the supervision of the PM which is configured to use a single storage (STS and LTS connected). $10 \mathrm{mF}$ has been chosen to enable a relatively fast cold start at low light and yet hold enough energy for transmission of SF7 frames.

- The SX1276 LoRa radio from Semtech is needed for LPWAN communication [23]. It is mounted on the mbed Shield module that holds the needed components and interfaces with the microcontroller board via an SPI interface [23]. The RF power delivered by the SX1276 is programmable. However, that power strongly varies with the supply voltage. The relation is not clearly explained in the manufacturer's documents. Using a spectrum analyser, about 6.4 $\mathrm{dBm}$ was measured at the RF output for a supply voltage of $2 \mathrm{~V}$ and with setting for $+14 \mathrm{dBm}$. Adding the antenna gain, leads to a node transmitting with about $+8 \mathrm{dBm}$.

- The system is controlled by the Apollo2 microcontroller manufactured by Ambiq [24]. That device includes a Cortex-M4 compatible CPU. It was chosen to enable low-energy computation. The microcontroller runs an adapted LoRaWAN stack that uses an external serial FRAM to store important parameters.

- The sensor is a BME680 from Bosh Sensortec [25]. It measures environmental parameters such as temperature, relative humidity, air pressure.

- A serial FRAM from Fujitsu [26] is added in order to save critical parameters that should not 
change in case of power loss. The FRAM technology is low power at read and write and enables a high number of read/write cycles (in the order of $10^{13}$ ), making it appropriate for applications where energy is in short supply.

- A voltage converter is used in order to keep the embedded system at a low voltage $(2 \mathrm{~V})$ while the supercapacitor is discharged into the load. A buck converter is appropriate here, since it is more efficient that an LDO.

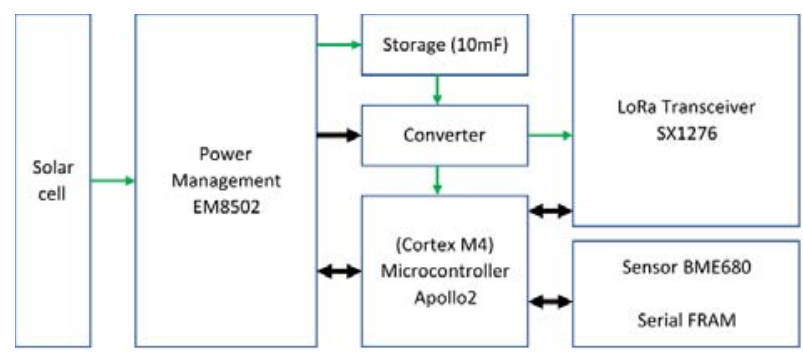

Figure 1. Block diagram of the node

\section{B. Low-power Strategy}

The EM8502 cannot deliver enough current to directly power the LoRa transceivers. For that reason, an external circuit (controlled by the PMIC) is necessary. The limits of the PMIC are set so that the system starts working when the voltage of the storage capacitor reaches $3 \mathrm{~V}$. It can then be discharged down to $2 \mathrm{~V}$. If there is enough energy from the solar cell, the supercapacitor is charged to a maximum of $3.6 \mathrm{~V}$. The energy available while discharging a $10 \mathrm{mF}$ supercapacitor from $3 \mathrm{~V}$ down to $2 \mathrm{~V}$ is $25 \mathrm{~mJ}\left(\left(3^{2}-2^{2}\right) * 5\right)$. The energy available between $3.6 \mathrm{~V}$ and $2 \mathrm{~V}$ is $44.8 \mathrm{~mJ}$. A load requiring $25 \mathrm{~mJ}$ or less can therefore be powered once the storage has reached 3 V. Selecting a supercapacitor with a higher capacitance allows more energy to be accumulated. The more energy is needed in the storage, the longer the charging process (at a given illuminance level). For instance, if a higher RF output power or a higher spreading factor is wanted. A supercapacitor of $100 \mathrm{mF}$ will hold 10 times the energy, once it is fully charged.

The node is designed to enable efficient harvesting and to allow processing and communication activities with small amount of energy. A central concept is the reduction of the load energy consumption during energy scavenging, in order to enable harvesting under low input power conditions (low light). To fulfil that, the embedded system is kept active only when necessary. Otherwise it should draw a minimal amount of energy. This is normally achieved by using the low-power modes of the microprocessor. In such modes, the processor is still powered, but draws little energy (tens of nA to several $\mu \mathrm{A})$. Leakages tend to increase with temperature, affecting the energy requirements. In this design, elements that are not needed are switched off. After the active work, important parameters are saved in the serial FRAM and the embedded system is completely turned off. Therefore, the energy consumption of the system during harvesting only depends on the power management consumption and the leakages of elements such as the supercapacitor. This allows the module to harvest efficiently, improving the low light performance. After a programmed time (10 minutes in this work), the device is restarted if there is enough energy available to do the work at hand. Critical parameters are read from the FRAM and stored in the microcontroller's RAM for further processing.

\section{SETUP, MEASUREMENTS, RESUltS AND DISCUSSION}

The setup elements are shown in Fig. 2 and Fig. 3.

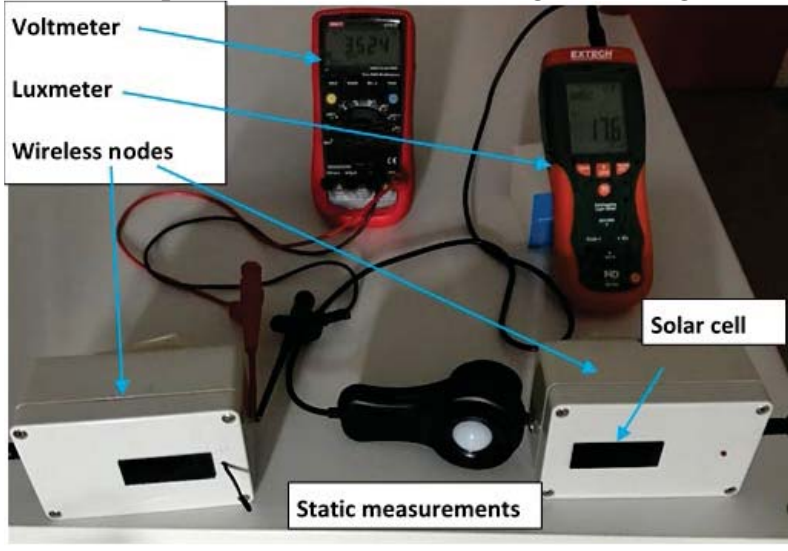

Figure 2. Solar nodes and measurement instruments

The system was tested in an office using artificial and natural light. Measurements were made in the afternoon, to take advantage of the natural illuminance reduction. Curtains were used to darken the room. Artificial light from the fluorescent lamps was employed to go above 100 lux when needed. In order to measure the illuminance, the HD450 [27] luxmeter was used. It has an own internal timer base.

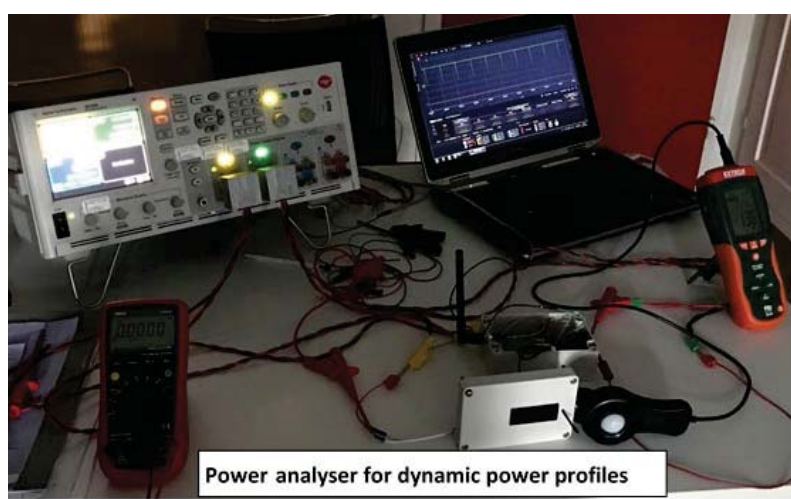

Figure 3. The power analyzer records dynamic profiles

The solar-powered node was programmed to make measurements and transmit every 10 minutes using the SF7BW125 spreading factor. The frame included information such as temperature, relative humidity, air 
pressure, status data of the power management (to give an idea of the relative input current and the storage voltage).

Several measurement runs were made in the course of this work. Those shown here are from the $17^{\text {th }}$ July 2020. A power analyzer was set up to monitor the voltage of the storage element and the current and voltage of the solar cell. To observe the data sent by the node, the infrastructure of the ZHAW-InES School of Engineering was put to task. LoRaWAN compatible gateways at different places in the building received the frames and forwarded the data to a server for logging. Different scripts extracted and displayed the needed information from the JSON files.

Fig. 4 shows the illuminance during the nearly 6 hours of the test. The small modulations on the signal reflect the movements of the curtains at the window and people in the room, making the setup more realistic.

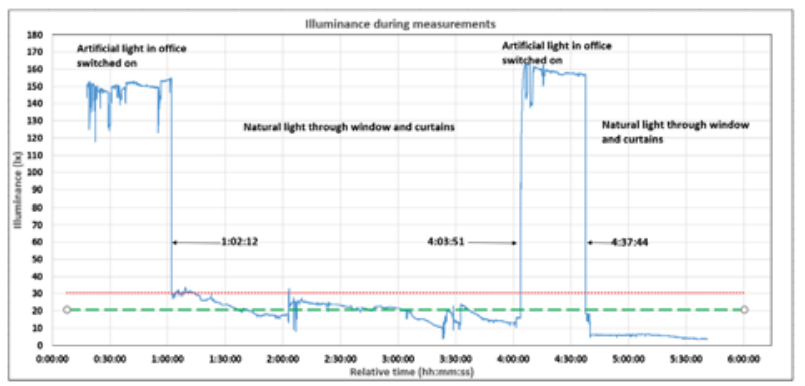

Figure 4. Illuminance during the measurements

Fig. 5 shows the storage's voltage and the solar cell's voltage. Relative time is used to allow an easy interpretation (instruments have different time bases).

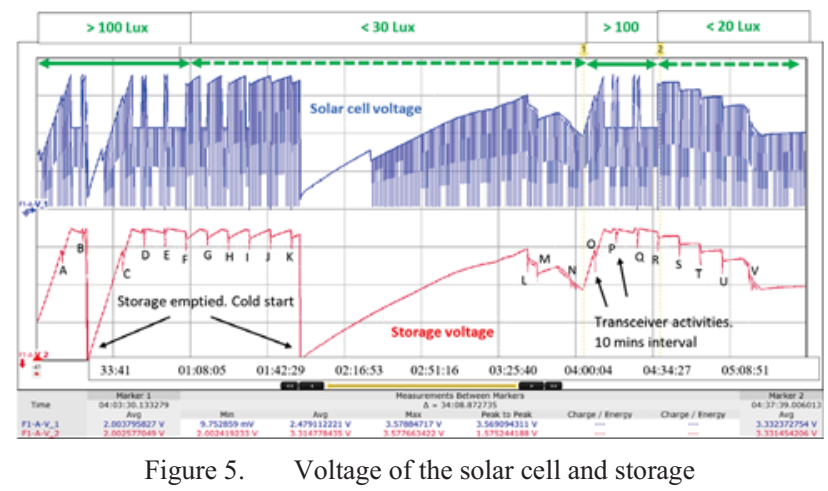

The light was switched on, triggering the harvesting process. The recording of the illuminance started a little later than the recording of current and voltage profiles. There are basically 4 zones in the measurements.

\section{A. First Zone}

During the first hour, artificial light was switched on, leading to an illuminance level above 100 lux. In that period, the node harvested fast and was soon able to go into active mode, measuring and transmitting every 10 minutes (time points A, B of Fig. 5). The storage was then emptied by short circuiting it, in order to simulate a cold start. 15 minutes later, the node was sending again, having scavenged enough energy to enable a reboot of the microcontroller (time points C, D, E, F of Fig. 5).

\section{B. Second Zone}

The artificial light was then switched off. The solar cell was only illuminated by light coming through the window and curtains (less than 30 lux) for about 3 hours. The node continued to harvest energy and to transmit every 10 minutes (time points G, H, I, J, K of Fig. 5). The storage was emptied again to emulate a cold start with the illuminance level around 30 lux. 100 minutes later, the system was active and sending again (time point L of Fig. 5). The curtain was closed, and the illuminance reduced. Below 20 lux, the harvested energy was no longer enough to keep the system active, leading to a shut down after the last transmission (time point $\mathrm{M}$ on Fig. 5).

\section{Third Zone}

The light was again switched on for about 30 mins. More than 100 lux of artificial light was available, leading to harvesting and transmitting (time points $\mathrm{O}, \mathrm{P}, \mathrm{Q}, \mathrm{R}$ of Fig. 5).

\section{Fourth Zone}

The light was switched off and the curtains tightened. Less than 20 lux illuminated the desk where the measurements were made. Measurements were taken for another hour, as the energy dwindled and the node went into shutdown (time points O, P, Q, R of Fig. 5).

Fig. 6 and Fig. 7 give more details on the system and on the measurements. Fig. 6 is a zoom on the last part of the measurement, where the illuminance value is below 20 lux. The storage energy is reduced in steps, each time that the node is activated (measure and transmit). The harvested energy is not enough to compensate for the losses. Once the low limit programmed in the comparator is reached, the system is shut down.

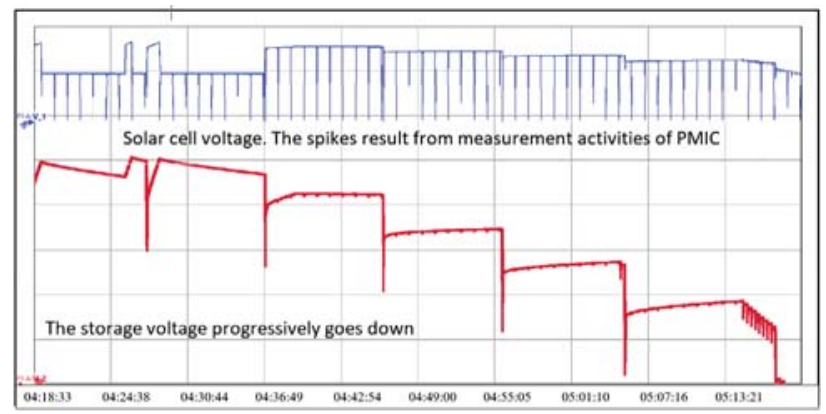

Figure 6. Zoom on the behaviour at 20 lux.

Fig. 7 is a zoom on the active part of the node. The peaks represent the rapid discharge of the storage element as the microprocessor is switched on, boots, makes measurements, initializes the radio, and transmits data. Only one receive window is used. That is shown by the second peak, when the receiver is switched on following a short time in low energy mode. The energy consumption 
can be estimated to $8 \mathrm{~mJ}$, based on the storage voltage before and after the communication activities (marker1/marker2 respectively at $3.48 \mathrm{~V}$ and $3.24 \mathrm{~V}$ ) and the $10 \mathrm{mF}$ storage value. With a storage voltage at $3.6 \mathrm{~V}$ (44 mJ available), the node can measure and transmit up to 5 times without any extra energy input. A storage of $100 \mathrm{mF}$ will allow the system to save enough energy for the transmission that require 100-200 $\mathrm{mJ}$ (SF11, SF12).

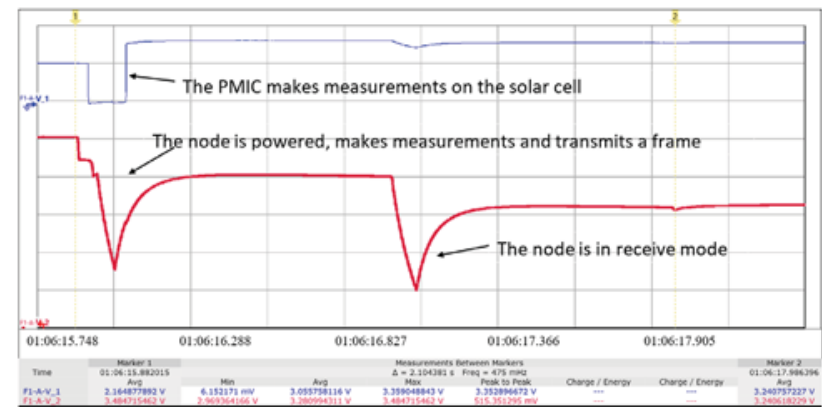

Figure 7. Zoom on active part of storage voltage.

Fig. 8 is a screenshot of the results from the MATLAB script used to visualize the frames received by the LoRaWAN gateways. Each dot represents a frame that has been received. The input current gives a relative indication of the energy that is been harvested. A 4-bit measurement in the PMIC is used to capture that input current. The storage voltage is indirectly deduced by using comparators set in the EM8502, and its value is also shown. Although the resolution does not allow for much granularity, the different illuminance periods can easily be recognized. The voltage variations on the storage can also be seen to match those shown on Fig. 5

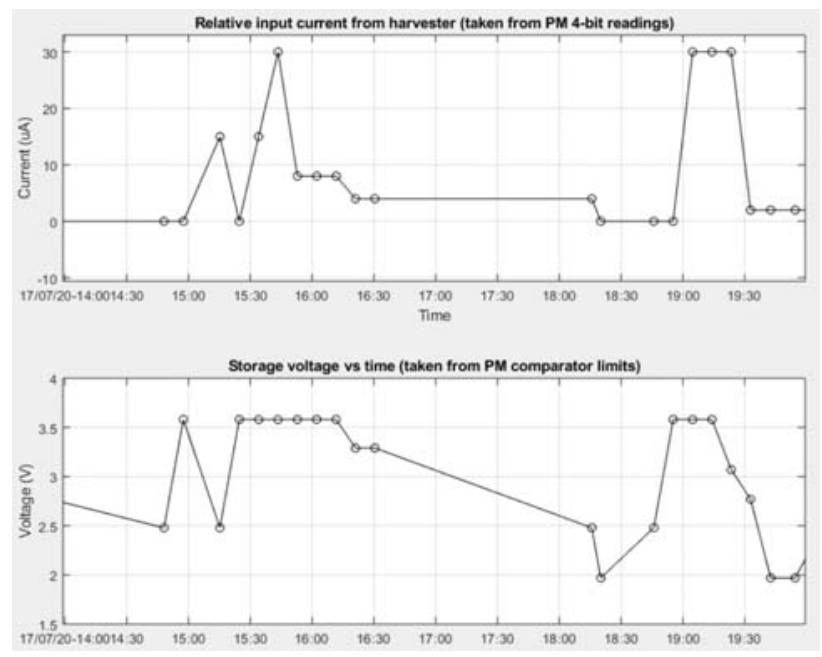

Figure 8. Frame reception and PM information. The circles indicate the time the frames were received.

Fig. 9 shows the temperature, relative humidity and air pressure, parameters measured by the BME680 sensor. The dots are placed at the measurement times and fit the transmission time points of Fig. 5 .

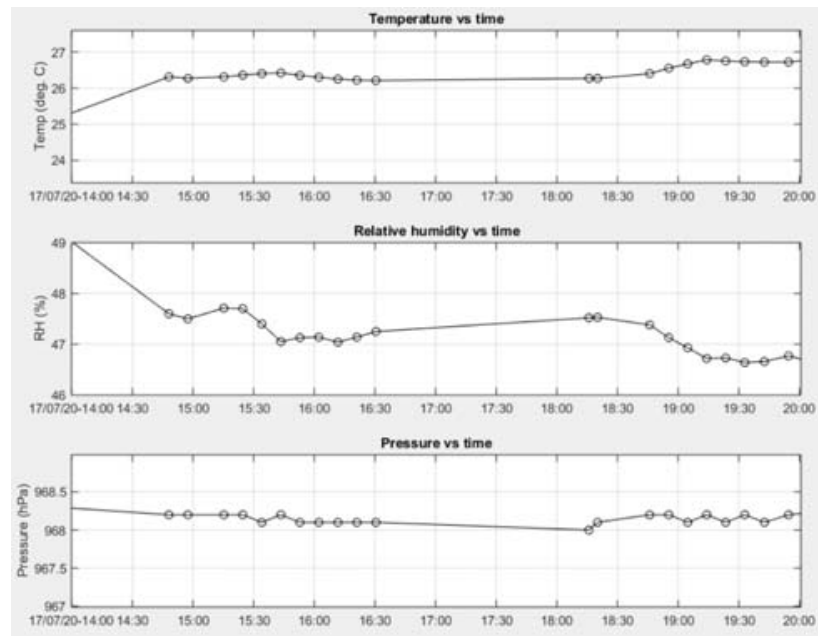

Figure 9. BME680 measurements. The circles indicate the time the frames were received.

One can deduce the following from the measurements:

- The system can be started when light intensity is under 30 lux and kept running with that illuminance level. The loss of energy due to the measurement and communication activities is more than compensated. The fact that several frames could be sent between the voltage limits of the PMIC means that one could also afford to use a load that requires $25 \mathrm{~mJ}$ with same components. This will for instance mean that transmission with a higher RF power or a higher spreading factor is possible, increasing the range of the system. The energy could also be used for more processing if that is required. It is important to get enough time to recharge the storage element.

- Between 30 lux and 20 lux, a system that is already started can be sustained.

- Below 20 lux, the system seems to have reached its limits. Further optimization is possible in order to reduce the illuminance needed for a sustainable activity.

\section{CONCLUSIONS AND FUTURE WORK}

This work has shown that, using new solar cells and carefully designed embedded systems, it is possible to run long range IoT nodes on harvested energy in an indoor environment under low-light conditions. 30 lux is enough to cold start the system and to sustain measurements and communication activities for a building automation application. A longer range can also be achieved with the proper compromises. With $8 \mathrm{~cm}^{2}$, the solar cell is small enough to allow small nodes to be built.

In future activities, the node will be optimized to allow the use of even smaller solar cells and to further reduce the illuminance limit. Measurements will also be reported for higher spreading factors. The new SX1261 device will be used instead of the SX1276 in order to transmit with more RF power at low supply voltages. The STS and LTS 
will be combined (or another PM used) to optimize startup and long-term storage of energy.

\section{REFERENCES}

[1] J.-P. Bardyn, T. Melly, O. Seller, and N. Sornin, "IoT: The era of LPWAN is starting now," in Proc. Conf. 42nd Eur. Solid-State Circuits Conf. (ESSCIRC), Lausanne, Switzerland, 2016, pp. 2530.

[2] K. Mekki, E. Bajic, F. Chaxel, and F. Meyer, "A comparative study of LPWAN technologies for large-scale IoT deployment," ICT Express, 2018, doi:10.1016/j.icte.2017.12.005.

[3] A.I. Petrariu, A. Lavric, and E. Coca, "Renewable energy powered LoRa-based IoT multi sensor node," 2019 IEEE 25th International Symposium for Design and Technology in Electronic Packaging (SIITME)

[4] M. Keshtgary, and A. Deljoo, "An efficient wireless sensor network for precision agriculture," Can. J. Multimedia Wireless Netw., vol. 3, no. 1,pp. 1-5, 2012.

[5] N. Wang, N. Zhang, and M. Whang, "Wireless sensors in agriculture and food industry-Recent development and future perspective," Comput.Electron. Agricult., vol. 50, pp. 1-14, Jan. 2006.

[6] J. Shah and B. Mishra, "IoT enabled environmental monitoring system for smart cities," 2016 International Conference on Internet of Things and Applications (IOTA), pp. 383-388, 2016, DOI: 10.1109/IOTA.2016.7562757

[7] W. Cong, L. Ji, Y. Yuanyuan, and Y. Fan, "Combining solar energy harvesting with wireless charging for hybrid wireless sensor networks," IEEE Transactions on Mobile Computing, vol. 17, no. 3, pp. 560-576, 2017, DOI: 10.1109/TMC.2017.2732979;

[8] W. Fan, J.-M. Redoute, and M. R. Yuce, "A self-powered wearable body sensor network system for safety applications", IEEE SENSORS, 2018, DOI: 10.1109/ICSENS.2018.8589848;

[9] P. Mars, and D. McIntosh, "Using a supercapacitor to power wireless nodes from a low power source such as a $3 \mathrm{~V}$ button battery," Sixth International Conference on Information Technology New Generations, 2009, DOI 10.1109/ITNG.2009.259;

[10] L. Joris, F. Dupont, P. Laurent, P. Bellier, S. Stoukatch, and J.-M. Redoute, "An autonomous Sigfox wireless sensor node for environmental monitoring," IEEE Sensors Letters, vol. 3, no. 7, 2019, DOI: 10.1109/LSENS.2019.2924058;

[11] M. Meli, and P. Bachmann. "Powering long range wireless nodes with harvested energy," $13^{\text {th }}$ Wireless Congress,Systems \& Applications, Munich, November 9-10, 2016

[12] W. Fan, J.-M. Redoute, and M. Yuce, "WE-Safe: A Self-Powered Wearable IoT Sensor Network for Safety Applications Based on LoRa,” IEEE Access. 1-1. 10.1109/ACCESS.2018.2859383.
[13] G. Loubet, A. Takacs, E. Gardner, A. De Luca, F. Udrea, and D. Dragomirescu, "LoRaWAN battery-free wireless sensors network designed for structural health monitoring in the construction domain," Sensors 2019, 19(7), 1510, DOI 10.3390/s19071510

[14] M. Meli, S. Stajic, and S. Wick, "Powering Sigfox nodes with harvested energy," Embedded World Conference 2020, Nürnberg, 25-27. Februar 2020

[15] R.J.M. Vullers, R.V. Schaijk, H.J. Visser, J. Penders, and C. Hoof, "Energy harvesting for autonomous wireless sensor networks," IEEE Journal of Solid-State Circuits, vol.2, no.2, pp.29-38, 2010.

[16] M. Mabon, M. Gautier, B. Vrigneau, M. Le Gentil, and O. Berder, "The smaller the better: Designing solar energy harvesting sensor nodes for long-range monitoring", Wireless Communications and Mobile Computing, Volume 2019, Hindawi, Article ID 2878545.

[17] M. A. Green, Y. Hishikawa, W. Warta et al., "Solar cell efficiency tables (version 50)," Wiley Journal of Progress in Photovoltaics:Research and Applications,vol.25, no.7, pp.668676, 2010.

[18] I. Mathews, S.N. Kantareddy, T. Buonassisi, and I.M. Peters, "Technology and market perspective for indoor photovoltaic cells", Joule, Volume 3, Issue 6, 19 June 2019, Pages 1415-1425

[19] M. Meli, and L. Hegetschweiler, "Affordable energy autonomous wireless sensor for day and night", Embedded World Conference, Nuremberg, Germany, 23-25 February 2016

[20] G. Peruzzi, and A. Pozzebon, "A review of energy harvesting techniques for Low Power Wide Area Networks (LPWANs)" Energies, 13 (3433), DOI 10.3390/en13133433, 2020.

[21] ExCellLight EXL10-4V170 module. [Online]. Available: https://lightricity.co.uk/excelllight-ex110-4v170 [Accessed: Sept. 1 st, 2020].

[22] EM8502- PMU \& DC Energy Harvesting Controller [Online]. Available: https://www.emmicroelectronic.com/product/pmu-dcenergy-harvesting-controller/em8502 [Accessed: Sept. $1^{\text {st }}, 2020$ ].

[23] Information on SX1276 LoRa transceiver and mbed Shield. [Online]. Available: https://www.semtech.com/products/wirelessrf/lora-transceivers/sx1276 [Accessed: Jul. 27, 2020].

[24] Apollo2 information from Ambiq [Online]. Available: https://ambiq.com/apollo2-low-power-microcontroller-family/ [Accessed: Sept. $\left.2^{\text {nd }}, 2020\right]$.

[25] Gas sensor BME680 [Online]. Available: https://www.boschsensortec.com/products/environmental-sensors/gas-sensorsbme680/ [Accessed: Sept. $\left.1^{\text {st }}, 2020\right]$.

[26] FRAM Technology \& Benefits [Online]. Available: https://www.fujitsu.com/uk/microsite/feeu/products/semiconducto rs/memory/fram/products/index.html [Accessed: Sept. $1^{\text {st }}, 2020$ ].

[27] HD450: Datalogging Heavy Duty Light Meter [Online]. Available: http://www.extech.com/products/HD450 [Accessed: Sept. 4, 2020] 